\title{
Perspectiva Intercultural de Género en la Radio Comunitaria de URACCAN, Recinto Bluefields
}

\author{
Gender Intercultural Perspective in URACCAN Community Radio, Bluefields Campus
}

Diana Marbely Oporta McMurray ${ }^{1}$

Ada Irania Villareal Alemán ${ }^{2}$

\section{Resumen}

El presente trabajo analiza la perspectiva intercultural de género en la Radio Comunitaria de URACCAN, en el recinto Bluefields, desde el abordaje y transmisión de los diferentes programas. El enfoque del estudio es cualitativo, con una metodología acción-participante en una estrategia articulada de tal forma que permitió generar saberes desde la visión de sabias y sabios quienes desde sus conocimientos y experiencias forjaron el debate propuesto. Entre los principales resultados, se consigue constatar que en Bluefields, se debe incidir de manera constante, en despejar las barreras y asegurar la participación de las mujeres en ámbitos donde tradicionalmente ha sido excluida. La perspectiva intercultural de género reconoce que la condición de subordinación de la mujer, se reproduce en la esfera pública producto de relaciones de poder imperantes, prácticas discriminatorias y ausencia de oportunidades. Finalmente, el estudio analiza el ejercicio de la interculturalidad y el género desde la radio con un llamado de la audiencia a no permitir que la emisora se convierta en un medio comercial, al contrario, que lo comunitario se fortalezca y que la radio sea de la comunidad y para la comunidad.

Palabras clave: Perspectiva intercultural de género; interculturalidad; radios comunitarias; sabios y sabias.

\section{Abstract}

The present document analyzes the gender intercultural perspective in the URACCAN Community Radio, in Bluefields campus, from the approach and transmission of the different programs. The focus of the study is qualitative, with an action-participant methodology in a strategy articulated in such a way that it allowed to generate knowledge from the vision of wise elderly people who from their knowledge and experiences forged the proposed debate. Among the main results, it is possible to confirm that in Bluefields, it is necessary to influence constantly, to clear the barriers and ensure the participation of women in areas where they have traditionally been

\footnotetext{
Máster en Comunicación Intercultural con Enfoque de Género y Docente de la carrera de Comunicación Intercultural del Recinto Bluefields. E-mail: dianaoporta@gmail.com - ORCID: https://orcid.org/oooo-ooo2-9977-5863

2 Máster en Docencia Universitaria. Tutora de la investigación. E-mail: raleman4@hotmail.com
}

Recibido: 26/02/2018 Aprobado: 28/08/2018 
excluded. The intercultural perspective of gender recognizes that the condition of women subordination is reproduced in the public sphere as a result of prevailing power relations, discriminatory practices and lack of opportunities. Finally, the study analyzes the exercise of interculturality and gender from the radio, with a call from the audience not to allow the station to become a commercial media, on the contrary, that the community be strengthened and that the radio be from the community and for the community.

Keywords: Gender Intercultural Perspective; Interculturality; Community Radios; Wise Elderly.

\section{Introducción}

La presente investigación se realizó en la Radio Universidad de URACCAN Recinto Bluefields en el periodo comprendido, enero a abril del 2016. Tiene como propósito aportar a la reflexión de la perspectiva intercultural de género incorporada en la programación de la radio y, analizar la incidencia que este medio ejerce en el buen vivir de la audiencia, en su mayoría de pueblos indígenas, afrodescendientes y mestizos. El estudio responde al problema de desigualdad existente entre hombres y mujeres y la violencia de género.

Busca que las mujeres sin importar su etnia o religión consigan formar parte de las decisiones que se toman y, que las políticas públicas que se dictan sean pensadas en ellas y las beneficie. Es un estudio que desde su análisis busca trascender a la mujer de espacios meramente familiares a públicos y de fortalecimiento de los pueblos.

Este trabajo también reflexiona sobre las acciones de incidencia promovidas por la universidad URACCAN para erradicar la desigualdad de género. De allí la importancia en centrar este estudio en la Radio Universidad (comunitaria intercultural), un medio que por excelencia resulta de fácil acceso. La experiencia nos llevó a identificar las necesidades de la audiencia, con quienes se articuló una nueva propuesta de programación.

\section{Revisión de literatura \\ Fundamentación teórica de las sabidurías y conocimientos}

\section{Perspectiva Intercultural de Género}

Para James (Comunicación personal, 19 de marzo 2016), la perspectiva intercultural de género se refiere a la cultura y costumbre de cada etnia, por medio de sus tradiciones culturales y su historia, podemos conocerlos más a fondo, respetarnos unos a los otros sin importar género o etnia. Es el respeto entre mujer y hombre sin distinción 
alguna, todos y todas tenemos los mismos derechos, no debemos de seguir más en la subordinación del más fuerte.

Para Gargallo (2012), la construcción de un sistema de género hegemónico, que descansa en la contraposición binaria entre lo femenino y lo masculino como dimensiones excluyentes y exageradamente diferenciadas, propia de las clases dominantes americanas que actúan en Abya Yala sobre la idea de dualidad. Abya Yala es el nombre dado a América por el pueblo Kuna de Panamá y Colombia antes de la llegada de Cristóbal Colón y los europeos. (Hernández, 2004)

Para Lareos (Comunicación personal, 21 de marzo 2016) con la creación del mundo inició la división y la diferencia entre el hombre y la mujer. No se declararon los mismos derechos para todos. De allí la importancia de enmarcar la armonía como parte esencial de la igualdad de derechos. (L. Omeir, Comunicación personal, 19 de marzo 2016).

\section{Comunicación Intercultural}

Desde la perspectiva de la Universidad de las Regiones Autónomas de la Costa Caribe Nicaragüens (URACCAN), la comunicación Intercultural se entiende como un proceso constante, dinámico y no exento de conflictos, que dota de sentido social, cultural, político e histórico las relaciones que establecen los actores sociales, mediante el intercambio y contraste de sus versiones con respeto de procesos o acontecimientos específicos (URACCAN, 2009).

De acuerdo a Omeir (comunicación personal, 19 de marzo 2016) la comunicación y la interculturalidad son dos conceptos que van de la mano, no se puede hablar de uno sin incluir al otro. La comunicación intercultural busca el respeto en las diferentes manifestaciones culturales, a través de lenguas en común que faciliten el diálogo, el reconocimiento de sí mismo y de los otros, así como intercambiar culturas y costumbres sin perder la propia.

Desde la perspectiva de Lareos (comunicación personal, 21 de marzo 2016), la lengua representa una parte fundamental para comprender la vida y la historia de los diferentes pueblos. Es muy primordial en la vida, en la narración de las historias colectivas de las comunidades étnicas, de los caminos que traspasaron en el transcurso del tiempo, de los aprendizajes obtenidos.

Sigue diciendo Lareos que la lengua materna es la que una persona aprende en su entorno familiar, mediante la cual se comunica normalmente, es la lengua propia. Con el empoderamiento la identidad de cada etnia permitirá que exista una comunicación más fluida en la ciudad afirma K. A. James (Comunicación personal, 19 de marzo 2016). 
La radio transforma la vida de los hombres y las mujeres de las comunidades. Anteriormente, los hombres y, sobre todo las mujeres no podían expresarse en un medio como la radio. Ahora es uno de los espacios vitales en donde las mujeres podemos dar a conocer nuestras inquietudes, propuestas y sueños. (Vásquez, 2012).

A pesar de la apertura de los medios comunitarios, son pocas las mujeres que han tenido acceso a estos, debido al machismo heredado del colonialismo, en donde las mujeres siempre se perciben en oposición a los hombres y en los que se asume implícitamente que los sistemas religiosos, legales, económicos, familiares, y comunitarios, son construidos por hombres. (Lareos, Comunicación personal, 2016).

La radio comunitaria promueve la defensa de los derechos humanos, una programación y una publicidad que interconecte a los oyentes, facilitándole los elementos que los otros medios difícilmente les ofrecen, siendo un canal abierto dejan los micrófonos para que se expresen libremente y compartan su vida, sus tropiezos, sus experiencias, alegrías y esperanzas.

\section{Materiales y métodos Caminos y formas del CCRISAC utilizados}

De esta manera, el presente estudio se focaliza en al propio contexto en que está inmersa la perspectiva intercultural de género y por ende la Radio Universidad en URACCAN recinto Bluefields. Esto significa que se trabajó desde la visión de las mujeres, mestizas, creoles, miskitas y rama que habitan en los barrios que sintonizan y escuchan la radio. Aprovecho para transparentar mi participación como colaboradora de la radio en el período 2012-2016.

El camino a seguir se sustenta en el enfoque cualitativo, utilizando el diálogo epistemológico, donde los saberes, conocimientos y prácticas de las diferentes etnias ocupan un lugar protagónico. La metodología acción participativa permitió generar conocimientos desde la visión de especialistas vinculados al campo de la comunicación. De esta manera, se aplicaron entrevistas, encuestas y el monitoreo a la programación de la radio en el período comprendido de enero a abril del año 2016.

Por lo anterior, este estudio retoma la lógica del cultivo y crianza de sabidurías y conocimientos (CCRISAC) como una propuesta bioética que parte de procesos colectivos desde y para los pueblos, nacionalidades y culturas, para fortalecer y revitalizar su autonomía, autodeterminación, identidad, sabidurías, expresiones, conocimientos, prácticas y episteme. (RUIICAY, 2015) 


\section{Resultados y Discusión \\ Sistematización del CCRISAC}

\section{Perspectiva Intercultural de Género en la Radio Universidad de URACCAN.}

La universidad URACCAN es una institución única en su modelo comunitario Intercultural, que trabaja con los distintos grupos étnicos e indígenas de la Costa Caribe de Nicaragua. Entre sus líneas estratégicas de funcionamiento la comunicación intercultural representa una vía importante de desarrollo y de visibilización de los pueblos, "la comunicación debe verse como el espacio necesario e ideal para establecer un diálogo interno que nos lleve a articular el quehacer de una institución", y desde el rol de la radio esta es "un vehículo indispensable para transmitir y compartir el conocimiento, con una función educativa, cultural, de esparcimiento, informativa y de formación del pensamiento de la comunidad".(Política de Comunicación Intercultural de URACCAN, 2009).

La historia de Radio Universidad inicia en el año 2012 cuando sus ondas hertzianas despertaron al pueblo del Caribe Sur. Su capacidad de transferencia y potencia es 1 kilowatts. Transmite en el 91.1 F.M del dial. Físicamente su torre está ubicada en el recinto URACCAN Bluefields, cuenta con un sistema de pararrayos y protección eléctrica. Además, con equipos de transmisión (transmisor, excitador, consola de audio, protector de corriente eléctrica, computadora y micrófonos), sala de controles y locución, sala de estudio de grabación de viñetas, programas y cuarto para el equipo transmisor.

La emisora, con seis aproximadamente de estar en el aire, se caracteriza por ser educativa, comunitaria, intercultural y de entretenimiento. Llega a comunidades urbanas y rurales del Caribe Sur. Entre el público de mayor audiencia y que reporta sintonía en la radio están jóvenes y mujeres. Sus contenidos se enfocan a temas de género, ambientales, deportivos, educativos, medicina tradicional, cultura y social.

La radio le contribuye a la sociedad transmitiendo eventos académicos, culturales, investigativos, de extensión social y comunitaria. Su prioridad se sustenta en el quehacer institucional, visibilizando eventos, efemérides, temas de actualidad, avances del modelo pedagógico, líneas estratégicas y campañas. De igual manera, construye contenidos distribuidos en distintos formatos radiofónicos (radio reportajes, viñetas, micros y noticieros, crónicas informativas, radio-revista, charlas, mesas redondas y cadenas radiales).

Los materiales radiofónicos, los contenidos, la musicalización y los mensajes, son realizados en lenguas propias de la región (español, creole y miskito), cumpliéndose en la práctica la perspectiva intercultural de género. Este esfuerzo incluye otros programas realizados en lengua materna. 
Radio Universidad también integra contenidos realizados por otras entidades en formatos atractivos y dinámicos entre los que destacan: 100 Mujeres en Conflicto (microprogramas), Tiempos de Huracanes (radionovelas), Dra. Miralles (Sexualidad), Medio ambiente, Derecho Consuetudinario. Estos programas ayudan a consolidar el modelo radial comunitario intercultural. Un aspecto que cuida la radio es la música, no se pautan aquellas canciones que promueven la violencia física ni psicológica, machistas, que empujan al suicidio, la discriminación étnica y de género.

Una de las variables importantes que plantea la radio es la integración de la Perspectiva Intercultural de Género, a través de sus contenidos, mensajes, música y capacitaciones brindados de forma periódica a sus controlistas y locutores/as, considerada esta estrategia un mecanismo certero que aporta a los cambios en la sociedad. Tiene el propósito de cambiar actitudes y comportamientos en los seres humanos, promover una vida justa, equitativa, libre de prejuicios y desigualdades.

Reconocer la Perspectiva Intercultural de Género es entender que día a día se está viviendo con desigualdades y que no se está reconociendo las propias perspectivas culturales de mujeres y hombres como parte constitutiva del la Autonomía de los Pueblos y el ejercicio efectivo de la ciudadanía Intercultural.

“(...) Las mujeres debemos de formar parte de un cambio que requiere esfuerzo para lograr la visibilización ante los hechos que han permanecido en las sociedades como la discriminación, opresión, violencia, machismo, esto da la pauta para lograr una vida diferente." Entrevista a James (Comunicación personal, 19 de marzo 2016).

Radio Universidad apunta a difundir temáticas que la población demanda, asi lo confirman un sondeo realizado en Bluefields donde se plantearon horarios, temas y contenidos por parte de los oyentes. De acuerdo a una de las entrevistadas, Villarreal (Comunicación personal, o8 de marzo 2016), esto se debe a que la radio es comunitaria Intercultural y se busca aportar a la educación diaria de la población oyente.

“(...) Las radios comunitarias deben de encaminarse a la construcción de espacios que brinden información relevante y actuales. Es necesario que se aproximen al contexto de las comunidades étnicas, siendo visible que la única radio en Bluefields que cuenta con contenido comunitario es Radio Universidad". Entrevista a Omeir (Comunicación personal, 19 de marzo 2016). 
Temas sugeridos por la población encuestada

\begin{tabular}{|c|c|}
\hline Clasificación & Temas \\
\hline Género & $\begin{array}{l}\text { 1. Mujeres que ocupan cargos de jefas en ONG'S e instituciones del gobiernos y } \\
\text { 2. } \text { violentan los derechos de sus subordinados (mujeres). } \\
\text { toma de decisiones. } \\
\text { 3. Mujeres del campo o del casco rural } \\
\text { 4. Mujeres florero: mito o realidad. } \\
\text { 5. Género e Interculturalidad en la educación } \\
\text { 6. Género e Interculturalidad en la sociedad o instituciones sociales } \\
\text { 7. Reconociendo las diferencias culturales, mediante la aplicación de las prácticas de } \\
\text { 8. Eénero. } \\
\text { 9. Políticas gubernamentales institucionales con respecto a las relaciones } \\
\text { 10. La importerculturales con enfoque de género. } \\
\text { intercultural de género } \\
\text { 11. Erradicación de la Violencia } \\
\text { 12. Interculturalidad comunitaria con enfoque de género. } \\
\text { 13. Respeto a la cultura, costumbres y diversidad. } \\
\text { 14. Ciudadanías interculturalidad de género. } \\
\text { 15. Vivir plenamente, sin perderle el respeto a Dios. } \\
\text { 16. Identidad (dignidad, igualdad y solidaridad). } \\
\text { 17. Cosmovisión étnica } \\
\text { 18. Derechos humanos } \\
\text { 19. Globalización } \\
\text { 20. Libertad de expresión } \\
\text { 21. Tolerancia } \\
\text { 22. Masculinidad emergente }\end{array}$ \\
\hline Salud & $\begin{array}{l}\text { 1. Sobre las enfermedades más comunes en la zona } \\
\text { 2. Prevención de las enfermedades. } \\
\text { 3. Apoyo a las campañas de vacunación que se llevan a cabo en la zona } \\
\text { 4. La limpieza y la salud } \\
\text { 5. Preservación, cuidado e incremento de las áreas verdes } \\
\text { 6. Nutrición, etc. }\end{array}$ \\
\hline $\begin{array}{c}\text { Integración y } \\
\text { consolidación de las } \\
\text { familias }\end{array}$ & $\begin{array}{l}\text { 1. Paternidad responsable } \\
\text { 2. Economía familiar } \\
\text { 3. Relación padre-hijos } \\
\text { 4. Problemas juveniles } \\
\text { 5. Revalorización de la mujer en la familia }\end{array}$ \\
\hline
\end{tabular}




\begin{tabular}{|c|ll|}
\hline Clasificación & \multicolumn{2}{|c|}{ Temas } \\
\hline & 1. & Funciones de las organizaciones \\
& 2. & Papel de los dirigentes \\
& 3. & Trabajos y luchas comunales \\
Organización de los & 4. & Valores humanos que se deben cultivar \\
pobladores & 5. & Historia de mi barrio \\
& 6. & Promoción del arte y la cultura popular \\
& 7. & Alentar la realización de festivales artísticos \\
& 8. & Difundir las experiencias culturales. \\
& 9. & Organizar concursos de poesía, música, teatro, pintura popular \\
\hline
\end{tabular}

Verificando el diálogo desde los y las encuestadas sobre el perfil de la radio, el $70 \%$ refiere que es una radio que combina lo comunitario con lo comercial. Un $28 \%$ expresa que es comunitario, porque aporta a la educación de la población, comparte contenidos educativos interculturales, reflexiona en temas sobre leyes y publicidades educativas. Solo el $2 \%$ asegura que no sabían, coincidiendo este planteamiento con lo mencionado por Omeir.

El 100\% de los encuestados propone que el perfil de la Radio Universidad responda a la educación intercultural y a transmitir el quehacer de la universidad de forma objetiva, aportando a la educación y abordando las dificultades contribuyendo de esta manera a la solución de problemas sociales. En este orden, una de las consultadas refiere que la radio es de perspectiva comunitaria pues sus programas son educativos e informan a la población.

Insisten en demandar que la radio permanezca como comunitaria, que se fortalezca y que no se convierta en una radio comercial, que sea una radio para la comunidad, (no amarillista ni sensacionalista) que les ayude a mejorar la calidad educativa y por ende su vida. Demandan a la a continuar aportando al empoderamiento de los derechos y deberes, para erradicar los estereotipos machistas.

Una de las entrevistas mencionó la necesidad de empoderar a las mujeres de bajos recursos económicos pidiendo a la sociedad "que no se les discrimine por esta, por ser negras, miskitas o de otra etnia, que el color de piel, que la forma del cuerpo o el estatus económico no impida que exista una igualdad o un cambio positivo que apunte a mejorar el buen vivir".

En relación a la programación, el 20\% de los encuestados asegura haber escuchado la radio en tres diferentes lenguas (español, creole, miskito), un 30\% solo creole, un $45 \%$ solo español y un $5 \%$ en todas las lenguas mencionadas. Expresan estar satisfechos porque la programación radial toma en cuenta los contextos interculturales presentes en la Costa Caribe Sur. 
Una de las entrevistas solicita ampliar la transmisión de los contenidos a más de tres lenguas y que se elaboren programas educativos en el tema de la interculturalidad.

“(...) deberían de tener programas de las diferentes etnias o tomar en cuenta a cada una, para poder impulsar la lengua propia, esto daría más confianza a la población para reportarse y hablar en su propia lengua." Expresión de una de las personas encuestadas.

El estudio evidencia que existe preocupación por el concepto de multiculturalidad que se está trabajando en la ciudad, dado que esta solo hace alusión a la coexistencia más no una hermandad, no se está viviendo en convivencia a como representa la interculturalidad, vivir con respeto e igualdad entre todas y cada una de las diferentes etnia.

En la radio universidad se busca como abordar los temas de fortalecimiento de las comunidades indígenas y afrodescendientes, con el fin de aportar al buen vivir y vivir bien de cada ciudadano, sin embargo hace falta promover la participación activa y el protagonismo de la comunidad.

Como parte de los hallazgos encontrados, la radio trata de abordar la Perspectiva Intercultural de Género, aportando de esta manera a revertir el aislamiento sociocultural que viven las mujeres. Radio Universidad llega hasta la comodidad de los hogares y lugares en donde no llegan los programas y organizaciones que trabajan temas de género e interculturalidad.

Las encuestas expresan que además de conceptualizar con programas pregrabados y viñetas la interculturalidad y género, se debe utilizar ejemplos muy claros de modo que sea entendido por todo público que escuche el programa (joven, adulto, letrado e iletrado).

“(...) Si nos invitan o brindan un espacio en la radio universidad como pueblo ramacreole con gusto lo aceptamos, e inclusive el tener un programa, así podremos compartir con nuestra familia indígena y afrodescendientes e impulsar programas para mejorar nuestras condiciones de vida." Expresión del presidente del territorio Rama-Creole.

\section{Incidencia de la Perspectiva Intercultural de Género desde Radio Universidad}

“(...) se les agradece mucho a los de radio universidad por su labor, porque con los programas de cien mujeres en conflicto me han ayudado a mejorar la relación familiar." Expresión de una de las mujeres protagonistas. 
La incidencia que la radio universidad ha tenido es calificado de manera positiva según la entrevista, porque les ha ayudado a revalorar su cultura, generando un nuevo territorio, mejores relaciones donde priman, la cotidianidad, ideas, creencias y prácticas ancestrales.

Vivimos en una región multilingüe y pluricultural en donde a diario nos relacionamos e intercambiamos y trabajamos con personas que tienen diferentes costumbres, culturas y pensamientos. Cada uno de los grupos étnicos e indígenas tiene sus propias tradiciones. La diversidad de costumbres, culturas y lengua debe verse como una riqueza y no como un motivo para distanciarse. Encuestado confirman que desde la Radio Universidad se ha inculcado el respeto a las diferentes etnias.

“(...) el respeto a la Interculturalidad, al género y a la diversidad de los pueblos étnicos de la Costa Caribe Sur, es cuando se logra el vivir libremente siempre y cuando tu libertad no dañe al otro." Expresión de una de las personas encuestadas.

Líderes del territorio Rama-Creole expresaron que es bueno que se les ayude, se les tome en cuenta y eduque por medio de la radio. Piden desarrollar más el tema de género e interculturalidad, y así erradicar el patriarcado de la comunidad.

\section{Estrategia Radio Universidad para incluir la Perspectiva Intercultural de Género}

Sostener y realizar un proyecto comunicacional como la radio debe verse desde varios ángulos como: organizacional, contenido, comunicacional y financiero, traducido desde lo que indica Lama (2002), nos dice que la gestión de una radio debe desarrollarse desde las dimensiones político cultural, comunicacional, organizacional y económica. La radio comunitaria y ciudadana se define por la comunidad de intereses compartidos que representa y por los objetivos político-culturales, organizacional comunicacionales y empresarios coherentes con esos mismos intereses.

Parámetros dimensionados que debe regir en una radio Lama (2002), para la gestión de la radio, no es únicamente hacer programas al aire o repartir tareas. Es un proceso que integre y articula una serie de dimensiones, entre lo político, cultural, comunicacional, organizacional y económico.

La gestión implica articular estas cuatro dimensiones, en el sentido de hacer crecer de la manera más paralela y simultanea posible. Lo más armoniosamente posible. Obviamente hay momentos en los cuales uno se dedica más a uno de estos aspectos que a los otros. Pero para que la radio sea fuerte y crezca, debe haber un desarrollo en equilibrio entre las cuatro dimensiones. En el caso de la Radio Universidad las dimensiones de mayor desarrollo han sido la comunicacional y el político cultural, entre los desafíos a mejorar las dimensiones organizacional y económica. 
Ilustración \#1. Reflexiones sobre las dimensiones de la radio universidad, su estado y propuesta.

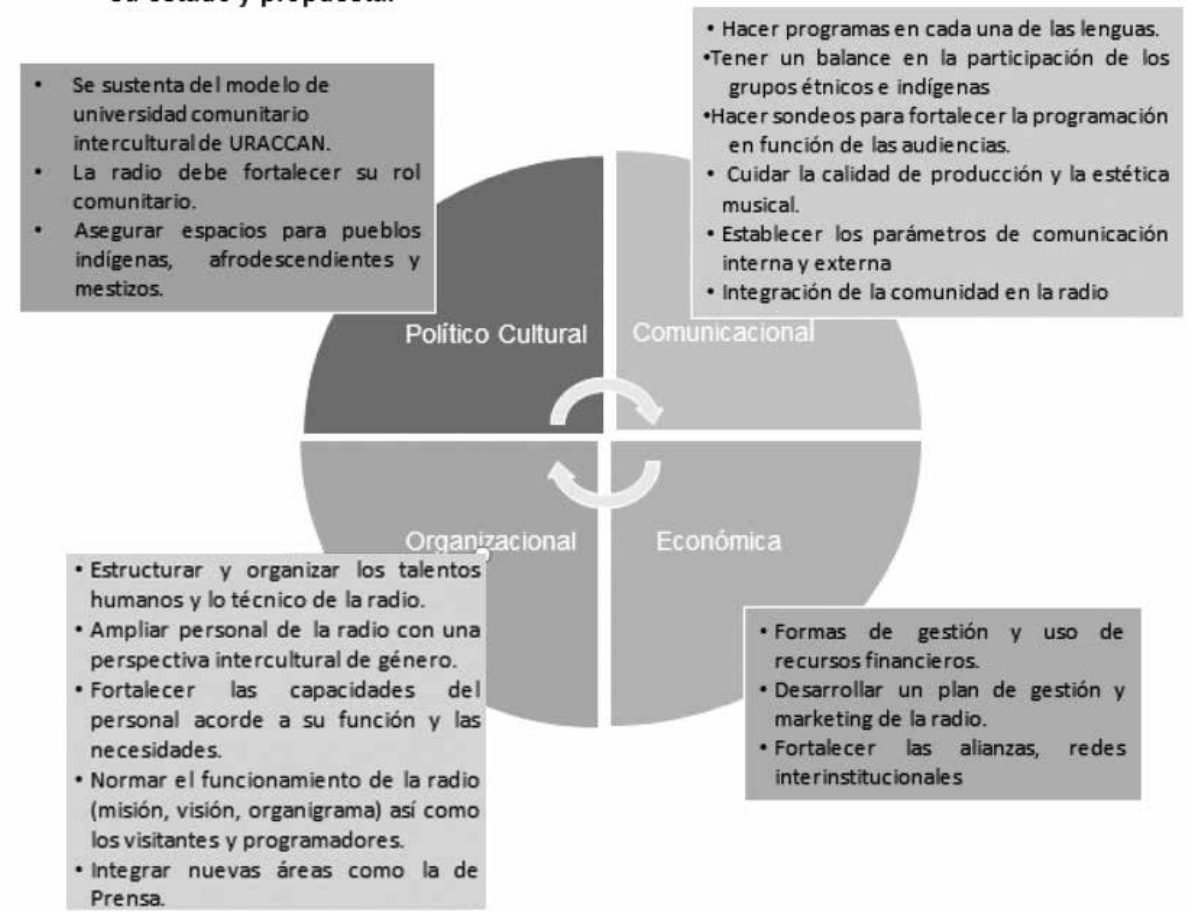

Elaboración propia.

\section{Conclusiones}

En la actualidad la radio universidad no cuenta con el financiamiento permanente de subvenciones públicas, ni de cuotas de asociados. Por ello, cuenta con poca publicidad y con el apoyo de otra área del Instituto de Comunicación Intercultural (ICI), que es la que complementa cada uno de los gastos de la radio. La publicidad no genera suficiente recursos económicos, por tal motivo la radio debe de buscar o enmarcar a obtener otras actividades que generen recursos económicos para su sustento, fortalecer las alianzas, redes inter-institucionales y se pueden realizar cursos de diferentes temáticas donde la radio se desempeñe mejor, ofertarlo a la población en general.

Hacer productos para venderlos ( $c d$ con combos musicales), ofertar servicios de edición y grabación de viñetas y programas, hasta algún temas en específico que la población requiera grabar. También ofertar programación especial por hora en la radio, generar espacios radiales a las instituciones y organizaciones. 
Animación de eventos, perifoneo, alquiler de audio y montar show en la ciudad trayendo a personalidades nacionales del gusto de la población que les permita generar más recursos económicos. Pero para poder realizar cada una de estas actividades se debe de garantizar los equipos necesarios.

Otro aspecto a considerar es las necesidades de formación del personal, este requiere fortalecer sus conocimientos capacidades en expresión oral, animación, uso de las nuevas tecnologías. Además se debe de impulsar la producción de programas interactivos en lo técnico y en la diversificación de voces (masculinas, femeninas en las lenguas de la región).

\section{Lista de referencias}

Gargallo, F. (2012). Diversas teorías y prácticas feministas de mujeres indígenas. Colombia.

Hernández, M.A. (2004). Encuentros en los senderos de Aby-Yala. Quito: Ediciones Abya Yala. Recuperado de: http://repository.unm.edu/bitstream/handle/1928/11484/ EncuentrosenlossenderosdeAbyayala.pdf?sequence $=1$

RUIICAY (2015). Documento base para el Cultivo y Crianza de Conocimientos y Sabidurias. Red de Universidades Indígenas Interculturales y Comunitarias de Abya Yala.

Lama E. (2002). Gestión radiofónica. Bolivia.

URACCAN (2009). Política de Comunicación Intercultural de la Universidad de las Regiones Autónomas de la Costa Caribe Nicaragüense (URACCAN).

Vásquez, G.C. (2012). Mujeres de la palabra florida: comunicando pensamientos en radio Jënpo. Chasqui. Revista Latinoamericana de Comunicación, (120), 9-13. 